\title{
Validation of Parenting Styles and Dimensions Questionnaire (PSDQ) on Adolescents
}

\author{
Siti Atiyyatul Fahiroh \\ Faculty of Psychology, Muhammadiyah University of Surabaya \\ atikfahiroh@gmail.com \\ Mareyke M.W. Tairas \\ Faculty of Psychology, Airlangga University \\ mareyke.tairas@psikologi.unair.ac.id \\ Sofia Retnowati \\ Faculty of Psychology, Gadjah Mada University \\ sofia_retnowati@yahoo.com
}

\begin{abstract}
The objective of this study is to determine the validity and reliability level of Parenting Styles and Dimensions Questionnaire (PSDQ) on adolescents in Senior High School of Surabaya city. Participants consisted of 204 students of Senior High School from several regions in Surabaya. They were between 16 and 19 years old. Confirmatory Factors Analysis (CFA) was used for the Parenting Styles and Dimensions Questionnaire (PSDQ) of Robinson, at all (1995). From 62 items in this measurement found 52 items that meet the validity requirements for father's parenting measurement and 52 items that meet the validity requirements for mother's parenting measurement.
\end{abstract}

Keywords: Parenting styles, psychometric validation, adolescence.

\section{Introduction}

The research of parenting style in adolescents is significant, and evolved for decade. Parents' attitudes and behavior towards children will directly influence the development of personality and temperament, and their mental health (Fu at al 2013). Improper parenting will cause misbehavior in children. The findings from Aminayi, at al. (20I5) showed that anxiety in children is strongly associated with parenting style and self-efficacy. 
Research conducted by Lansford et al. (2004) from a representative community sample of 585 children followed from five-year-old kindergarten to IIth grade found that mothers who reported the use of physical discipline in the first five years of their child's during 6th grade on II years and in the 8th grade on 13 years, present various externalizing negative behaviors including aggression, violence, and school problems and problems with the police. The results of this research indicate that three out of seven children showed externalizing behavior problems. This result demonstrates that used violence in parenting will cause externalizing problems in further years.

Milevsky et al. (2008) examined maternal and paternal parenting and the variation of adolescents' well-being as a function of a combination of parenting styles. Participants in the previous study of 272 students in grades 9 and II of a public high school in a large city in the northeast of the United States. The most common parenting combination researched are of neglectful, or truly affectionate, mother and father. Participants with authoritative parenting style shows higher wellbeing compared to those brought up with a non-authoritative parenting style. Individuals whose parents, or at least one of them, uses an authoritative parenting style are more likely to be successful in achieving great wellbeing compared to the counterpart. Additionally, participants with both non-permissive parents, and with a non-permissive mother, exhibit lower self-esteem score compared to those with non-permissive fathers. In other words, we can conclude that parenting style can influence a child's self-esteem.

According to Baumrind (2010), compliance and conformity are characteristic of authoritarian parenting styles. Such parents will always expect that the rules in the home must be obeyed and implemented without any rejection and reason. Evidence of authoritarian families is found in Caucasian teenage families. They show poor social skills, low levels of self esteem and high levels of depression (Milevsky, et al., 2008).

Unlike the authoritarian family, permissive family parenting is more likely to be characterized by a high level of responsiveness, but a low level of demand (Baumrind, 
at al, 20l0). Permissive parents behave more affirmatively or encourage/convince the impulses and actions of children when making decisions. They do not set rules and coercion to avoid being involved in behavioral control and necessity in the actions of their teenage children. Therefore, permissive families will show a decrease in monitoring their children after they reach adolescence so that behavior affected by the outside world increases (Luyckx et al. 20l I). The effect of this is the behavior of drug users, wrong behavior in schools, and less positive orientation towards higher and higher schools. According to Ginsburg \& Bronstein (1993), permissive parenting is also associated with low self-esteem and extrinsic motivational orientation among adolescents.

What about good parenting? Which parenting style will produce responsible adolescents? According to Baumrind's theory in McMurty (2013), good or healthy parenting is demonstrated from appropriate parental behavior, one of which is through mature verbal expression to facilitate parent and child communication. With appropriate standards of behavior, parents become role models for their children, influencing the formation of healthy aspirations and increasing the independence of children. The child must continue to be educated with good patterns. Therefore, parenting in adolescents needs to be applied in families. Based on this, there needs to be validation of parenting measurement tools in accordance with the Indonesian cultural context. This will help the pattern of care for teenagers in Indonesia.

Previous research on parenting measurement was conducted by $\mathrm{Fu}$, et al (2013). Fu and colleagues conducted a study with the aim of evaluating the validity and reliability of Chinese version of PSDQ (Parenting Styles and Dimensions Questionnaire, PSDQ) by translating PSDQ into Chinese. The study was conducted on 443 selected parents of children aged 6-16 years who reside in Chongqing. Of these parents, 52 parents were chosen for intensive testing. They were retested for 6 weeks to assess the reliability of the retest. Determination of reliability is included in the internal consistency of parents. This is used to calculate Cronbach's coefficient. Reliability coefficient at the time of retest is done to calculate the correlation of the results in 
each subscale at each measurement period. We conduct content validity, structural validity, and confirmatory factor analysis to determine the validity of the measuring instrument. The results of the study indicate that the Parenting Style and Dimensions Questionnaire (PSDQ), in line with psychometric requirements, has good reliability and validity. In other words, it is useful as a tool for evaluating parenting style.

Initially, the questionnaire from this study consisted of 133 items in a 5-point Likert scale, ranging from I (Never) to 5 (Always). This questionnaire was developed using 80 items from the block report and 53 new items. The childcare practice questionnaire was completed by 1,251 volunteer parents (534 fathers and 717 mothers) who live in communities located in Utah. Of these participants, 32\% were parents of preschool-aged children from universities/Head Start Preschools and found 68\% were parents of schoolage children from parishes and public elementary schools. The average age of fathers who filled out was 37.9 years, with an age range of 22 to 63 . On the other hand, the average age of mothers who filled out the questionnaire was 35.6 years old, with an age range starting from 2 to 57 . The the average number of fathers has completed schools in 15.3 years (starting from 8 to 23), while mothers have completed schools for at least 14.6 years in average (from 8 to 23). The majority of study participants were Caucasians from two family countries with an average family income of around $\$ 30,000$. The number of questions retained was 62 items: 27 authoritative items $(\alpha=.91), 20$ authoritarian items $(\alpha=.86)$, and I5 permissive items $(\alpha=.75)$. Among the 62 items, 19 items (31\%) were from the Block report while 43 items were new (69\%).

Another study by Ismaili (2015) also used the adapting version of 58-items PSDQ. Ismaili study involved parents of children ages from 4 to 12 years. Cronbach's alpha and Pearson correlation analysis were used for the subscales of each parenting style scale. The results showed that reasoning/induction and democratic participation (legitimate parenting style) have Cronbach's alpha, $\alpha=.740$ and $\alpha=.655$ respectively. The dimensions of authoritarian parenting style and dimensions of permissive parenting style did not reach $\alpha=.70$ (the coefficient is equal to $r=.39$ and $r=.5$. $P<.0 \mathrm{I}$ ). 
This research aims to examine the validity and reliability of the Indonesian version of the Parenting Styles and Dimensions Questionnaire (PSDQ), which was originally constructed by Robinson, et al. (1995). This research is important, considering the origin culture of Robinson's PSDQ and colleagues are different from the culture of the current research place, Surabaya city, Indonesia.

This is in accordance with the opinion of Bornstein (2012), who stated that parenting is influenced by culture, with each culture having its own parenting characteristics. This happens because of the existence of ideas that are deeply rooted in feeling, thinking, and behaving in each culture. Furthermore, according to Bornstein (2012) crosscultural studies show that a behavior in one culture is normative, but in other cultures it is not. Indonesian family culture, especially Surabaya, is certainly different from the original culture of Robinson's PSDQ, therefore it is necessary to test the validity and reliability. The current research involved teenagers, and adapting original 62-items PSDQ. All three studies from Fu, et al (2013), Robinson, et al (1995), and Ismaili (20I5) have shown quite high validity and reliability.

\section{Method}

\section{Participants}

Participants of this study consist of senior high school students in Surabaya, East Java, Indonesia, which were also referred to as populations. According to Sugiyono (2015), in the research experienced limited funds, energy and time, when using all the subjects of the population can use the sample. The sampling technique used in this research was random. Random sampling technique or simple random sampling is the taking of sample members from the population done randomly without interpreting the strata that exist in that population. This way is done if members of the population are considered homogeneous. The characteristics of the subjects in this research were adolescents who received high school education in class XI and XII in Surabaya city. 204 Senior High School students aged 16-19 years. 


\section{Questionnaire}

This questionnaire is distributed in the classroom with the help of Guidance and counseling teachers, students, were looked for classes that can fill the scale of research calmly. Before this questionnaire was distributed, the participants were informed about the purpose of the research. They also agreed to fill out a questionnaire and sign an informed consent where the authors promised to maintain the confidentiality of the answers according to the standards of the research code of ethics.

The questionnaire that will be validated in this research is the Parenting Styles and Dimensions Questionnaire (PSDQ) of Baumrind's global parenting three dimensions that appear consistent with typology (I) authoritative (2) authoritarian, and (3) permissive. 62 items were evaluated using five points. This questionnaire was used to determine the perceptions of adolescents about their mothers and fathers parenting. Three parenting styles, authoritative parenting, authoritarian parenting and permissive parenting were further divided into II factors (dimensions). Authoritative parenting included 27 items which were divided into 4 factors: warmth \& involvement, reasoning/induction, democratic participation and good natured/easy going. Authoritarian parenting included 20 items which were divided into 4 factors: verbal hostility, corporal punishment, non-reasoning, punitive strategies and directiveness. Permissive parenting included I5 items (among which, 3 items with reverse scoring) which were divided into 3 factors: lack of follow through, ignoring misbehavior and self-confidence.

In this research the original Parenting Styles and Dimensions Questionnaire (PSDQ) from Robinson, at all (1995) was translated into Indonesian by researchers, then translated back into English by expert. It turns out that there is a conformity of meaning between the original questionnaire and the translation from Indonesian into English. Because the original questionnaire was addressed to parents as fillers of questionnaires, while by researchers this was intended as a perception of adolescents towards their parents then a statement was changed by the author. For example, in item no I, "Knows the names of child's friends" becomes "Ibu saya mengenal nama- 
nama teman-teman saya" (My mother knows the names of my friends), or "Ayah saya mengenal nama-nama teman-teman saya" (My father knows the names of my friends).

\section{Data Analysis}

The validity of this research is called construct validity, which is the type of validity that shows the extent to which the measurement tool reveals the theoretical constructs measured by validity requires statistical analysis techniques (Azwar 20I0).

The validity in this research was tested using confirmatory factor analysis or CFA. Confirmatory factor analysis is used to test whether a construct has unity professionally or whether the indicators used can confirm a construct or variable. If each indicator is a gauge of the construct indicator, it will have a high factor loading value. With confirmatory factor analysis researchers can test whether indicators are construct indicators. Confirmatory factor analysis will group each indicator into several factors (Ghozali, 20II). Modeling results with confirmatory factor analysis (CFA) or a full model with structural equation modeling (SEM) interpretation will be found from factor loading. By definition, loading factor is a large correlation between the indicator and the latent construct. According to Ferdinand (2005) testing lambda values (significance of the value of loading factors) in confirmatory factor analysis is done to see whether the variables used have sufficient meaning to define the latent variables formed. Statistical analysis using the AMOS version 20 program, the main objective of conducting confirmatory factor analysis is to test the model, so the most important part of the analysis is to test the fit of the model.

\section{Results}

The results of confirmatory factor analysis from Parenting Style and Dimensions Questionnaire (PSDQ) adapted from Robinson's et al PSDQ (1995) showed that not all items from 62 items are valid and reliable. There are 10 items in the data processing stage that do not meet the validity requirements (loading factor is less than .50 ) on father's parenting and 10 items on mother's parenting. Thus, there are 52 items in each 
father's parenting and mother's parenting that meet the requirements. Some items of the Parenting Styles and Dimensions Questionnaire (PSDQ) that did not meet these requirements were deleted, and re-analyzed. The results of Confirmatory Factor Analysis (CFA) can be seen in table I below.

Table I

Confirmatory Factor Analysis of father's and mother's parenting subscale of PSDQ in Early stage

\begin{tabular}{|c|c|c|c|c|c|c|c|c|c|c|c|}
\hline $\begin{array}{l}\text { Default } \\
\text { Model }\end{array}$ & $\mathrm{Cmin}$ & $\mathrm{df}$ & $P$ & Cmindf & GFI & AGFI & RMSEA & RMR & $\mathrm{CFI}$ & TLI & $\mathrm{NFI}$ \\
\hline $\begin{array}{l}\text { Father's } \\
\text { authoritative }\end{array}$ & 613.375 & 32 & 0.000 & 1.919 & .822 & .789 & .670 & .780 & .874 & .862 & .771 \\
\hline $\begin{array}{l}\text { Father's } \\
\text { authoritarian }\end{array}$ & 421.147 & 166 & 0.000 & 2.537 & .839 & .77I & .87I & .118 & .861 & .840 & .792 \\
\hline $\begin{array}{l}\text { Father's } \\
\text { permissive }\end{array}$ & 303.082 & 87 & 0.000 & 3.484 & .821 & .754 & 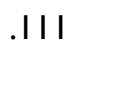 & .139 & .774 & .727 & .714 \\
\hline $\begin{array}{l}\text { Mother's } \\
\text { authoritative }\end{array}$ & 708.272 & 320 & 0.000 & 2.213 & .792 & .754 & .077 & .059 & .885 & .874 & .810 \\
\hline $\begin{array}{l}\text { Mother's } \\
\text { authoritarian }\end{array}$ & 408.950 & 166 & 0.000 & 2.464 & .822 & .775 & .085 & .106 & .859 & .839 & .786 \\
\hline $\begin{array}{l}\text { Mother's } \\
\text { permissive }\end{array}$ & 314.109 & 87 & 0.000 & 3.610 & .817 & .748 & .113 & .124 & .777 & .731 & .721 \\
\hline
\end{tabular}

Table 2

Confirmatory Factor Analysis of father's and mother's parenting subscale of PSDQin Second Stage

\begin{tabular}{|c|c|c|c|c|c|c|c|c|c|c|c|}
\hline Default Model & Cmin & $\mathrm{df}$ & $p$ & Cmindf & GFI & AGFI & RMSEA & RMR & CFI & TLI & $\mathrm{NFI}$ \\
\hline $\begin{array}{l}\text { Father's } \\
\text { authoritative }\end{array}$ & 543.3677 & 271 & 0.000 & 2.005 & 828 & 794 & .070 & .077 & .878 & .865 & .786 \\
\hline $\begin{array}{l}\text { Father's } \\
\text { authoritarian }\end{array}$ & 229.484 & 115 & 0.000 & 1.996 & .887 & .849 & .070 & .890 & .930 & .917 & .871 \\
\hline Father's permissive & 102.196 & 32 & 0.000 & 3.934 & .902 & .832 & .104 & .103 & .911 & .875 & .877 \\
\hline $\begin{array}{l}\text { Mother's } \\
\text { authoritarian }\end{array}$ & 206.347 & 86 & 0.000 & 2.388 & .879 & .931 & .082 & .064 & .920 & .902 & .871 \\
\hline $\begin{array}{l}\text { Mother's } \\
\text { permissive }\end{array}$ & 97.446 & 32 & 0.000 & 3.045 & .910 & .845 & .100 & .087 & .916 & .881 & .881 \\
\hline
\end{tabular}

The results of the Confirmatory Factor Analysis of the second stage Parenting Style and Dimensions Questionnaire (PSDQ) mother's parenting can be seen in table 3 below 
Table 3

The result of Confirmatory Factor Analysis on mother's parenting in second stage

\begin{tabular}{|c|c|c|c|c|c|c|}
\hline \multicolumn{7}{|c|}{ Authoritative Items } \\
\hline Items & M & SD & Load & AVE & CR & Factor I (Warmth \& Involvement) \\
\hline I & 3.81 & .978 & 0.608 & 0.706 & 0.916 & Ibu saya mengenal nama-nama teman-teman saya. \\
\hline 2 & 3.71 & .971 & 0.596 & & & $\begin{array}{l}\text { Ibu saya menyadari masalah atau yang menjadi pusat } \\
\text { perhatian saya di sekolah. }\end{array}$ \\
\hline 3 & 3.87 & .999 & 0.745 & & & $\begin{array}{l}\text { Ibu saya memberi pengahargaan kepada saya ketika saya } \\
\text { menjadi anak yang baik. }\end{array}$ \\
\hline 4 & 3.96 & .994 & 0.734 & & & $\begin{array}{l}\text { Ibu saya memberikan kenyamanan dan pemahaman ketika } \\
\text { saya sedang kecewa. }\end{array}$ \\
\hline 5 & 3.61 & 1.113 & 0.654 & & & $\begin{array}{l}\text { Ibu saya mengekspresikan afeksi melalui memeluk, } \\
\text { mencium dan menggendong anak. }\end{array}$ \\
\hline 6 & 3.98 & .955 & 0.82 & & & Ibu menunjukkan simpati ketika saya terluka atau frustasi. \\
\hline 7 & 3.83 & .998 & 0.782 & & & $\begin{array}{l}\text { Ibu saya sering mengapresiasi apa yang telah saya coba } \\
\text { atau tugas yang saya selesaikan. }\end{array}$ \\
\hline 8 & 4.13 & .842 & 0.693 & & & Ibu saya responsif terhadap perasaan atau kebutuhan anak. \\
\hline 9 & 3.78 & 1.079 & 0.729 & & & $\begin{array}{l}\text { Ibu saya mendorong saya untuk membicarakan kesulitan } \\
\text { saya, }\end{array}$ \\
\hline 10 & 3.82 & 1.133 & 0.694 & & & Ibu memiliki kehangatan dan keintiman dengan saya. \\
\hline II & 3.60 & 1.144 & 0.681 & & & $\begin{array}{l}\text { Ibu meminta maaf kepada saya ketika membuat suatu } \\
\text { kesalahan dalam pengasuhan. }\end{array}$ \\
\hline
\end{tabular}

Factor 2 (Reasoning/Induction)

\begin{tabular}{|c|c|c|c|c|c|c|}
\hline 12 & 4.00 & .897 & 0.742 & 0.776 & 0.913 & Ibu menjelaskan kepada saya akibat dari perilaku saya. \\
\hline 13 & 4.11 & .800 & 0.868 & & & Ibu memberikan alasan mengapa saya harus patuh. \\
\hline 14 & 3.81 & .879 & 0.696 & & & Ibu menekankan alasan-alasan tentang aturan yang ada. \\
\hline 15 & 4.01 & .849 & 0.777 & & & $\begin{array}{l}\text { Ibu saya membantu saya untuk memahami dampak dari } \\
\text { perilaku melalui memberi dukungan terhadap saya. }\end{array}$ \\
\hline 16 & 4.08 & .813 & 0.811 & & & $\begin{array}{l}\text { Ibu saya menjelaskan bagaimana kita merasakan perilaku } \\
\text { baik dan buruk. }\end{array}$ \\
\hline 17 & 3.94 & .903 & 0.798 & & & $\begin{array}{l}\text { Ibu membicarakan dengan saya lebih lanjut dan alasan- } \\
\text { alasannya ketika saya berperilaku salah. }\end{array}$ \\
\hline 18 & 3.86 & .883 & 0.728 & & & $\begin{array}{l}\text { Ibu saya memberitahu perilaku yang diharapkan anak } \\
\text { sebelum saya terlibat dalam aktivitas. }\end{array}$ \\
\hline \multicolumn{7}{|c|}{ Factor 3 (Democratic Participation) } \\
\hline 19 & 3.46 & 1.109 & 0.713 & 0.675 & 0.804 & $\begin{array}{l}\text { Ibu saya memperhitungkan pilihan pilihan saya dalam } \\
\text { membuat rencana keluarga. }\end{array}$ \\
\hline 20 & 3.47 & 1.055 & 0.813 & & & $\begin{array}{l}\text { Ibu saya mengijinkan saya untuk memberi masukan dalam } \\
\text { aturan keluarga. }\end{array}$ \\
\hline 21 & 3.71 & .950 & 0.623 & & & $\begin{array}{l}\text { Ibu saya memperhitungkan keinginan saya sebelum } \\
\text { meminta saya melakukan sesuatu. }\end{array}$ \\
\hline 22 & 3.23 & 1.121 & 0.667 & & & $\begin{array}{l}\text { Ibu mendorong saya mengekspresikan diri secara bebas } \\
\text { bahkan ketika saya tidak setuju kepada orang tua. }\end{array}$ \\
\hline 23 & 3.02 & 1.176 & 0.528 & & & $\begin{array}{l}\text { Ibu menyalurkan perilaku saya yang buruk ke dalam } \\
\text { aktivitas yang dapat diterima. }\end{array}$ \\
\hline
\end{tabular}




\begin{tabular}{|c|c|c|c|c|c|c|}
\hline \multicolumn{7}{|c|}{ Factor 4 (Good Natured/Easy Going) } \\
\hline 24 & 3.97 & .920 & 0.722 & 0.772 & 0.855 & Ibu mudah dan rileks dengan saya. \\
\hline 25 & 4.06 & .948 & 0.812 & & & Ibu menunjukkan kesabaran kepada saya. \\
\hline 26 & 4.03 & .928 & 0.725 & & & Ibu saya senang bercanda dan bermain dengan saya \\
\hline 27 & 3.78 & 1.023 & 0.824 & & & $\begin{array}{l}\text { Ibu saya menunjukkan penghargaan terhadap pendapat- } \\
\text { pendapat saya melalui mendorong saya untuk berekspresi. }\end{array}$ \\
\hline \multicolumn{7}{|c|}{ Authoritarian Items } \\
\hline Items & M & SD & Load & AVE & CR & Factor I (Verbal Hostility) \\
\hline 28 & 2.89 & 1.183 & 0.776 & 0.760 & 0.845 & Ibu saya sering meledakan kemarahan terhadap saya. \\
\hline 29 & 2.91 & 1.205 & 0.753 & & & Ibu saya berteriak ketika saya berperilaku salah. \\
\hline 30 & 2.72 & 1.118 & 0.793 & & & Ibu saya sering bertengkar dengan saya. \\
\hline 31 & 2.79 & 1.068 & 0.715 & & & Ibu saya sering berselisih dengan saya \\
\hline
\end{tabular}

\begin{tabular}{|c|c|c|c|c|c|c|}
\hline \multicolumn{7}{|c|}{ Factor 2 (Corporal Punishment) } \\
\hline 32 & 2.11 & 1.030 & 0.762 & 0.771 & 0.880 & $\begin{array}{l}\text { lbu menggunakan hukuman fisik sebagai cara } \\
\text { memdisiplinkan anak. }\end{array}$ \\
\hline 33 & 1.89 & .979 & 0.805 & & & Ibu memukul (pantat) saya ketika saya tidak patuh. \\
\hline 34 & 1.89 & 979 & 0.837 & & & Ibu menampar saya ketika saya berperilaku salah. \\
\hline 36 & 2.20 & 1.050 & 0.732 & & & $\begin{array}{l}\text { Ibu saya membimbing saya dengan hukuman berlebihan } \\
\text { daripada dengan alasan. }\end{array}$ \\
\hline 37 & 1.97 & .965 & 0.714 & & & Ibu menyigkirkan anak ketika anak itu tidak patuh. \\
\hline 38 & 2.48 & 1.149 & 0.73 & 0.720 & 0.762 & $\begin{array}{l}\text { Ibu menghukum saya dengan mengambil hak saya dengan } \\
\text { sedikit penjelasan. }\end{array}$ \\
\hline 39 & 2.00 & 1.022 & 0.8 & & & $\begin{array}{l}\text { Ibu saya menghukum dengan menempatkan saya di suatu } \\
\text { tempat sendirian dengan sedikit penjelasan. }\end{array}$ \\
\hline 40 & 2.39 & 1.190 & 0.62 & & & $\begin{array}{l}\text { Ibu saya menggunakan ancaman sebagai hukuman dengan } \\
\text { sedikit atau tidak ada pembenaran. }\end{array}$ \\
\hline \multicolumn{7}{|c|}{ Factor 4 (Directiveness) } \\
\hline 44 & 3.77 & 1.005 & 0.625 & 0.751 & 0.792 & $\begin{array}{l}\text { Ibu saya selalu memberitahu kepada saya apa yang harus } \\
\text { saya lakukan. }\end{array}$ \\
\hline 46 & 3.75 & .952 & 0.753 & & & Ibu saya menegur dan mengkritik untuk peningkatan saya. \\
\hline 47 & 3.81 & .912 & 0.856 & & & $\begin{array}{l}\text { Ibu saya menegur dan mengkritik ketika perilaku saya } \\
\text { tidak sesuai dengan harapan. }\end{array}$ \\
\hline \multicolumn{7}{|c|}{ Permissive Items } \\
\hline Items & M & SD & Load & AVE & CR & Factor I (Lack of Follow Through) \\
\hline 48 & 3.12 & & 0.438 & 0.541 & 0.621 & $\begin{array}{l}\text { Ibu menyatakan kepada saya akan menghukum saya dan } \\
\text { tidak benar-benar dilakukan. }\end{array}$ \\
\hline 49 & 2.69 & & 0.571 & & & $\begin{array}{l}\text { Ibu lebih sering mengancam saya dengan hukuman } \\
\text { daripada yang diberikan. }\end{array}$ \\
\hline 51 & 2.66 & & 0.55 & & & $\begin{array}{l}\text { Ibu menyerahkan kepada anak ketika anak menyebabkan } \\
\text { suatu keributan. }\end{array}$ \\
\hline 53 & 2.37 & & 0.593 & & & Ibu saya menyuap saya supaya saya patuh. \\
\hline \multicolumn{7}{|c|}{ Factor 2 (Ignoring Misbehavior) } \\
\hline 54 & 1.73 & & 0.93 & 0.849 & 0.883 & Ibu membiarkan saya mengganggu orang lain. \\
\hline 55 & 1.77 & & 0.928 & & & Ibu membiarkan saya sebagai penyebab orang lain marah. \\
\hline 56 & 1.89 & & 0.659 & & & Ibu mengabaikan saya perilaku salah. \\
\hline
\end{tabular}


Factor 3 (Self Confidence)

\begin{tabular}{|c|c|c|c|c|c|}
\hline 59 & 2,69 & 0.751 & 0.717 & 0.760 & $\begin{array}{l}\text { Ibu saya tidak yakin tentang cara mengatasi perilaku saya } \\
\text { yang salah. }\end{array}$ \\
\hline 60 & 2,61 & 0.74 & & & Ibu saya merasa sulit mendisiplinkan saya. \\
\hline 62 & 2,44 & 0.657 & & & $\begin{array}{l}\text { Ibu takut mendisiplinkankan anak karena anak takut } \\
\text { dikatakan kelakuannya buruk oleh anak yang menyebabkan } \\
\text { anak tidak menyukainya. }\end{array}$ \\
\hline
\end{tabular}

The results of the Confirmatory Factor Analysis on the second stage of Parenting Style and Dimensions Questionnaire (PSDQ) father's Parenting can be seen in table 4 below

\section{Table 4}

The result of Confirmatory Factor Analysis on father's parenting in second stage

\begin{tabular}{|c|c|c|c|c|c|c|}
\hline \multicolumn{7}{|c|}{ Authoritative Items } \\
\hline Items & $M$ & SD & Load & AVE & CR & Factor I (Warmth \& Involvement) \\
\hline 2 & 3.17 & 1.103 & 0.504 & 0.648 & 0.878 & $\begin{array}{l}\text { Ayah saya menyadari masalah atau yang menjadi pusat } \\
\text { perhatian saya di sekolah. }\end{array}$ \\
\hline 3 & 3.60 & 1.233 & 0.652 & & & $\begin{array}{l}\text { Ayah saya memberi pengahargaan kepada saya ketika saya } \\
\text { menjadi anak yang baik. }\end{array}$ \\
\hline 4 & 3.58 & 1.191 & 0.72 & & & $\begin{array}{l}\text { Ayah saya memberikan kenyamanan dan pemahaman ketika } \\
\text { saya sedang kecewa. }\end{array}$ \\
\hline 5 & 2.85 & 1.243 & 0.589 & & & $\begin{array}{l}\text { Ayah saya mengekspresikan afeksi melalui memeluk, mencium } \\
\text { dan menggendong anak. }\end{array}$ \\
\hline 6 & 3.68 & 1.120 & 0.657 & & & Ayah menunjukkan simpati ketika saya terluka atau frustasi. \\
\hline 7 & 3.58 & 1.109 & 0.745 & & & $\begin{array}{l}\text { Ayah saya sering mengapresiasi apa yang telah saya coba atau } \\
\text { tugas yang saya selesaikan. }\end{array}$ \\
\hline 8 & 3.90 & .998 & 0.678 & & & Ayah saya responsif terhadap perasaan atau kebutuhan anak. \\
\hline 9 & 3.30 & 1.111 & 0.612 & & & Ayah saya mendorong saya untuk membicarakan kesulitan saya, \\
\hline 10 & 3.18 & 1.128 & 0.641 & & & Ayah memiliki kehangatan dan keintiman dengan saya. \\
\hline II & 3.18 & 1.182 & 0.652 & & & $\begin{array}{l}\text { Ayah meminta maaf kepada saya ketika membuat suatu } \\
\text { kesalahan dalam pengasuhan. }\end{array}$ \\
\hline \multicolumn{7}{|c|}{ Factor 2 (Reasoning/Induction) } \\
\hline 12 & 4.02 & .915 & 0.653 & 0.712 & 0.877 & Ayah menjelaskan kepada saya akibat dari perilaku saya. \\
\hline 13 & 4.28 & .785 & 0.739 & & & Ayah memberikan alasan mengapa saya harus patuh. \\
\hline 14 & 3.83 & .948 & 0.612 & & & Ayah menekankan alasan-alasan tentang aturan yang ada. \\
\hline 15 & 4.03 & .865 & 0.731 & & & $\begin{array}{l}\text { Ayah saya membantu saya untuk memahami dampak dari } \\
\text { perilaku melalui memberi dukungan terhadap saya. }\end{array}$ \\
\hline 16 & 4.17 & .862 & 0.800 & & & $\begin{array}{l}\text { Ayah saya menjelaskan bagaimana kita merasakan perilaku baik } \\
\text { dan buruk. }\end{array}$ \\
\hline 17 & 3.92 & .887 & 0.699 & & & $\begin{array}{l}\text { Ayah membicarakan dengan saya lebih lanjut dan alasan- } \\
\text { alasannya ketika saya berperilaku salah. }\end{array}$ \\
\hline 18 & 3.84 & .961 & 0.735 & & & $\begin{array}{l}\text { Ayah memberitahu perilaku saya yang diharapkannya sebelum } \\
\text { saya terlibat dalam aktivitas. }\end{array}$ \\
\hline
\end{tabular}




\begin{tabular}{|c|c|c|c|c|c|c|}
\hline \multicolumn{7}{|c|}{ Factor 3 (Democratic Participation) } \\
\hline 19 & 3.47 & 1.038 & 0.702 & 0.661 & 0.754 & $\begin{array}{l}\text { Ayah saya memperhitungkan pilihan pilihan saya dalam } \\
\text { membuat rencana keluarga. }\end{array}$ \\
\hline 20 & 3.29 & 1.163 & 0.707 & & & $\begin{array}{l}\text { Ayah saya mengijinkan saya untuk memberi masukan dalam } \\
\text { aturan keluarga. }\end{array}$ \\
\hline 21 & 3.54 & 1.043 & 0.684 & & & $\begin{array}{l}\text { Ayah saya memperhitungkan keinginan saya sebelum meminta } \\
\text { saya melakukan sesuatu. }\end{array}$ \\
\hline 22 & 3.16 & 1.094 & 0.537 & & & $\begin{array}{l}\text { Ayah mendorong saya mengekspresikan diri secara bebas } \\
\text { bahkan ketika saya tidak setuju kepada orang tua. }\end{array}$ \\
\hline \multicolumn{7}{|c|}{ Factor 4 (Good Natured/Easy Going) } \\
\hline 24 & 3.89 & .937 & 0.718 & 0.707 & 0.798 & Ayah mudah dan rileks dengan saya. \\
\hline 25 & 4.10 & 1.022 & 0.645 & & & Ayah menunjukkan kesabaran kepada saya. \\
\hline 26 & 3.77 & 1.096 & 0.81 & & & Ayah saya senang bercanda dan bermain dengan saya. \\
\hline 27 & 3.58 & 1.040 & 0.642 & & & $\begin{array}{l}\text { Ayah saya menunjukkan penghargaan terhadap pendapat- } \\
\text { pendapat saya melalui mendorong saya untuk berekspresi. }\end{array}$ \\
\hline \multicolumn{7}{|c|}{ Authoritarian Items } \\
\hline Items & M & SD & Load & AVE & CR & Factor I (Verbal Hostility) \\
\hline & & & & 0.784 & 0.864 & \\
\hline 28 & 2.71 & 1.124 & 0.798 & & & Ayah saya sering meledakan kemarahan terhadap saya. \\
\hline 29 & 2.73 & 1.163 & 0.69 & & & Ayah saya berteriak ketika saya berperilaku salah. \\
\hline 30 & 2.45 & 1.128 & 0.69 & & & Ayah saya sering bertengkar dengan saya. \\
\hline 31 & 2.65 & 1.145 & 0.757 & & & Ayah saya sering berselisih dengan saya \\
\hline
\end{tabular}

\begin{tabular}{|c|c|c|c|c|c|}
\hline \multicolumn{6}{|c|}{ Factor 2 (Corporal Punishment) } \\
\hline 32 & 2.11 & 1.212 & 0.772 & 0.754 & $\begin{array}{l}\text { Ayah menggunakan hukuman fisik sebagai cara memdisiplinkan } \\
\text { anak. }\end{array}$ \\
\hline 33 & 1.80 & 1.037 & 0.838 & & Ayah memukul (pantat) saya ketika saya tidak patuh. \\
\hline 34 & $\mathrm{I}, 82$ & $\mathrm{I}, 077$ & 0.865 & & Ayah menampar saya ketika saya berperilaku salah. \\
\hline 35 & 2.75 & 1.229 & 0.503 & & Ayah meraih saya ketika saat saya tidak taat. \\
\hline 36 & 1.99 & 1.062 & 0.788 & & $\begin{array}{l}\text { Ayah saya membimbing saya dengan hukuman berlebihan } \\
\text { daripada dengan alasan. }\end{array}$ \\
\hline 37 & 1.83 & 1.008 & 0.702 & & Ayah menyigkirkan anak ketika anak itu tidak patuh. \\
\hline \multicolumn{6}{|c|}{ Factor 3 (Non-Reasoning, Punitive Strategies) } \\
\hline 38 & 2.31 & 1.190 & 2,31 & 0.702 & $\begin{array}{l}\text { Ayah menghukum saya dengan mengambil hak saya dengan } \\
\text { sedikit penjelasan. }\end{array}$ \\
\hline 39 & 1.83 & .968 & 0.757 & & $\begin{array}{l}\text { Ayah saya menghukum dengan menempatkan saya di suatu } \\
\text { tempat sendirian dengan sedikit penjelasan. }\end{array}$ \\
\hline 40 & 2.19 & 1.173 & 0.679 & & $\begin{array}{l}\text { Ayah saya menggunakan ancaman sebagai hukuman dengan } \\
\text { sedikit atau tidak ada pembenaran. }\end{array}$ \\
\hline \multicolumn{6}{|c|}{ Factor 4 (Directiveness) } \\
\hline 44 & 3.69 & 1.031 & 0.569 & & $\begin{array}{l}\text { Ayah menghukum saya dengan mengambil hak saya dengan } \\
\text { sedikit penjelasan. }\end{array}$ \\
\hline 45 & 3.13 & 1.084 & 0.646 & & $\begin{array}{l}\text { Ayah saya menghukum dengan menempatkan saya di suatu } \\
\text { tempat sendirian dengan sedikit penjelasan. }\end{array}$ \\
\hline 46 & 3.68 & 1.137 & 0.778 & & $\begin{array}{l}\text { Ayah saya menggunakan ancaman sebagai hukuman dengan } \\
\text { sedikit atau tidak ada pembenaran. }\end{array}$ \\
\hline 47 & 3.75 & 1.071 & 0.739 & & Ayah menghukum saya dengan mengambil hak saya dengan \\
\hline
\end{tabular}




\begin{tabular}{|c|c|c|c|c|c|c|}
\hline & & & & & & sedikit penjelasan. \\
\hline \multicolumn{7}{|c|}{ Permissive Items } \\
\hline Items & M & SD & Load & AVE & CR & Factor I (Lack of Follow Through) \\
\hline 48 & 2.98 & 1.172 & 0.76 & 0.672 & 0.672 & $\begin{array}{l}\text { Ayah menyatakan kepada saya akan menghukum saya dan } \\
\text { tidak benar-benar dilakukan. }\end{array}$ \\
\hline 49 & 2.58 & 1.152 & 0.811 & & & $\begin{array}{l}\text { Ayah lebih sering mengancam saya dengan hukuman daripada } \\
\text { yang diberikan. }\end{array}$ \\
\hline 51 & 2.71 & 1.042 & 0.547 & & & $\begin{array}{l}\text { Ayah menyerahkan kepada anak ketika anak menyebabkan } \\
\text { suatu keributan. }\end{array}$ \\
\hline 53 & 2.31 & 1.135 & 0.522 & & & Ayah saya menyuap saya supaya saya patuh. \\
\hline \multicolumn{7}{|c|}{ Factor 2 (Ignoring Misbehavior) } \\
\hline 54 & 1.75 & .936 & 0.928 & 0.829 & 0.865 & Ayah membiarkan saya mengganggu orang lain. \\
\hline 55 & 1.74 & .934 & 0.91 & & & Ayah membiarkan saya sebagai penyebab orang lain marah.. \\
\hline 56 & 1.79 & .961 & 0.61 & & & Ayah mengabaikan perilaku saya yang salah. \\
\hline \multicolumn{7}{|c|}{ Factor 3 (Self Confidence) } \\
\hline 59 & 2.58 & .925 & 0.796 & 0.708 & 0.746 & $\begin{array}{l}\text { Ayah saya tidak yakin tentang cara mengatasi perilaku saya yang } \\
\text { salah. }\end{array}$ \\
\hline 60 & 2.62 & 1.069 & 0.752 & & & Ayah saya merasa sulit mendisiplinkan saya. \\
\hline 62 & 2.39 & 1.176 & 0.551 & & & $\begin{array}{l}\text { Ayah takut mendisiplinkankan anak karena takut dikatakan } \\
\text { kelakuannya buruk oleh anak yang menyebabkan anak tidak } \\
\text { menyukainya. }\end{array}$ \\
\hline
\end{tabular}

\section{Discussion}

The results of Confirmatory Factor Analysis (CFA) above shows that the validity and reliability of Parenting Styles and Dimensions Questionnaires (PSDQ) are quite satisfying. This can be seen in the results of the Goodness of Fit test (the model test) that authoritative father's parenting subscale is moderate in Relative Chi square CminDF, and fit on the RMSEA and Comparative Fit Index (CFI). The results of construct reliability (CR) and variance extracted from the construct of father's authoritative parenting subscale test show construct reliability $(C R)$ values of $0.66 \mathrm{I}$ to 0.712 , variance extracted (AVE) 0.754 to 0.98 . This indicates that the reliability of each factor is acceptable.

Goodness of Fit test (Model test) results showed that authoritarian father's parenting subscale is fit on Relative Chi square CminDF, RMSEA, Comparative Fit Index (CFI) and Tucker-Lewis Index (TLI) and moderate on Goodness of Index (GFI), Adjusted Goodness of Index (AGFI), RMR and Tucker-Lewis Index (TLI). Construct Reliability 
$(C R)$ and Variance extracted (AVE) results from the construct of father's authoritarian parenting subscale test show Construct Reliability (CR) values are 0.688 to 0.784 , Variance extracted (AVE) is 0.743 to 0.885 , indicating that the reliability of each factor is acceptable.

Goodness of Fit test (Model test) result of permissive father's parenting subscale are fit on the Goodness of Index (GFI) and Comparative Fit Index (CFI) and moderate on the Adjusted Goodness of Index (AGFI). The results of Construct Reliability (CR) and Variance Extracted (AVE) of construct of father's permissible parenting subscale test show that construct reliability $(C R)$ is 0.672 to 0.829 , variance extracted (AVE) is 0.6 I to 0.865 , indicating that reliability of each factor is acceptable.

The results of the Goodness of Fit test (Model test) authoritative mother's parenting subscale are moderate on RMR, Comparative Fit Index (CFI), Tucker-Lewis Index (TLI) and NFI. The results of Construct Reliability (CR) and the Variance Extracted (AVE) of the construct of mother's authoritative parenting subscale test indicate that Construct Reliability (CR) value is 0.675 to 0.776 , Variance Extracted (AVE) is .855 to 0.916 . this show that reliability of each factor is acceptable.

The results of Goodness of Fit test (Model test) authoritarian mother's parenting subscale are fit on Comparative Fit Index (CFI) and Tucker-Lewis Index (TLI) and moderate on Goodness of Index (GFI), Adjusted Goodness of Index (AGFI), RMSEA, RMR and NFI. The results of Construct Reliability (CR) and Variance Extracted (AVE) of the construct of mother's authoritarian parenting subscale test show Construct Reliability (CR) values are .720 to $.77 \mathrm{I}$, Variance extracted (AVE) are .762 to .88. this indicates that the reliability of each factor is acceptable.

The results of Goodness of Fit test (Model test) permissive mother's parenting subscale are fit in the Goodness of Index (GFI) and Comparative Fit Index (CFI), and moderate on RMR, Tucker-Lewis Index (TLI), and NFI. Results of Construct Reliability $(\mathrm{CR})$ and Variance Extracted (AVE) of construct of mother's permissive parenting 
subscale test shows that Construct Reliability (CR) is .54I to .849 , Variance Extracted (AVE) is .621 to .883 , this indicates that the reliability of each factor is acceptable. Overall, it can be said that the adaptation of Parenting Style and Dimensions Questionnaire (PSDQ) from Robinson at all (1995) adequately fulfills the requirements when it is used as a measurement of the perception of adolescents about parenting in the Surabaya area.

Some items that do not meet the validity requirements (loading factor $<.50$ ) on authoritative father's parenting variable, on factor I (warmth \& involvement) are items number I and on Factor 2 (democratic participation item number 22). Statement in item number I is "Ayah saya mengenal nama-nama teman-teman saya" (My father know the names of my friends). It is possible because it is not father's habit, or it is not important for fathers, to recognize the names of their children's friends in the subject of this research. Statement on item number 22 is "Ayah mendorong saya mengekspresikan diri secara bebas bahkan ketika saya tidak setuju kepada orang tua" (Father encouraged me to express myself freely even when I disagree with parents). This is possible because in Indonesian culture a father does not usually encourage his children to express themselves freely.

Some items that do not meet the validity requirements (loading factor $<.50$ ) on the authoritarian father's parenting variable are in factor 3 (non-reasoning, punitive strategies) items number 4I, 42 and 43. Item number 4I, "Ketika ada dua anak yang sedang bertengkar, maka ayah akan memberikan disiplin ke pada anak yang lebih tua setelah itu baru kemudian mengajukan pertanyaan" (When there are two children who are quarreling, then the father will give discipline to the older child after which he then asks the questions) the sentence in this statement is difficult for the respondent to be understood, this also happens to the authoritarian mother's parenting variable. Item number 42, "Ayah saya lebih Nampak berhubungan dengan perasaannya sendiri dari pada perasaan anak" (My father seems more related to his own feelings than to the feelings of the child), and number 43, "Ketika saya bertanya kepada ayah mengapa saya harus menyesuaikan diri, menyatakan: karena saya adalah orang tuamu dan saya ingin kamu untuk 
melakukan" (When I asked daddy why I have to conform, he stated: because I am your parent and I want you to). Statement on item number 42 and number 43, both on authoritarian father's parenting and authoritarian mother's parenting do not meet the validity requirements (loading factor $<.50$ ), this may also be due to the participant's family who do not having the habit as stated in item number 42 and 43.

According to Bornstein (2012), each culture has differences from one another, which is caused by deep-rooted ideas related to feeling, thinking, and behaving in different cultures. Furthermore, according to Bornstein (20I2), culture is a form of different beliefs and behaviors between one group and another to organize daily life. This also applies to parenting style, where cultural differences will affect it.

Some items at permissive father's parenting, which do not meet the validity requirements (loading factor <.50) are found in factor I (lack of follow through) in item number 48, 49, 50, and 52, on factor 2 (ignoring misbehavior) in items number 57, 58, and 6I. Item number 48 "Ayah menyatakan kepada saya akan menghukum saya dan tidak benar-benar dilakukan" (Father stated to me that he will punish me and it is not actuall $y$ do them), item number 49 "Ayah lebih sering mengancam saya dengan hukuman dari pada yang diberikan" (Father threatens me more often with punishment than given) Item number 50 "Ayah memanjakan saya" (Father spoils me). Item number 52 "Ayah melakukan disiplin setelah saya melakukan perilaku salah" (Father does the discipline after I do wrong behavior). Item number 57 "Ayah menahan omelan dan/atau kritik bahkan ketika saya bertindak bertentangan dengan keinginan" (Father held back scolding and/or criticism even when I acted against his wishes.). Item number 58 "Ayah percaya diri tentang kemampuannya mengasuh saya" (Father is confident about his parenting abilities) and item number 6I "Ayah saya menetapkan aturan yang ketat untuk saya" (My father sets strict rules for me).

All dimensions on the authoritative mother's parenting variable are fulfilling the validity requirements (loading factor $>.50$ ). Some items that do not meet the validity requirements (loading factor $<.50$ ) in the authoritarian mother's parenting dimension 
are factor 3 (non-reasoning, punitive strategies), namely items number 35, 4I, 42, and 43 and factor 4 (directiveness), namely items number 45. Item number 35, "Ibu meraih saya ketika saat saya tidak taat" (Mom grabs me when I am disobedient). Item number 4I, "Ketika ada dua anak yang sedang bertengkar, maka ibu akan memberikan disiplin kepada anak yang lebih tua setelah itu baru kemudian mengajukan pertanyaan pertanyaan pada anak-anak berikutnya" (When there are two children who are fighting, the mother will give discipline to the older child afterwards and then ask questions to the next children). Statements of items number 35 and 41 may not be suitable for measuring parenting to reveal the authoritarian mother's parenting. Item number 43, "Ketika saya bertanya kepada ibu mengapa saya harus menyesuaikan diri, menyatakan: karena saya adalah orang tuamu dan saya ingin kamu untuk melakukan" (When I asked mom why I have to adjust, she said: because I am your parent and I want you to do it). Item number 45, "Ibu saya menuntut saya untuk melakukan sesuatu" (My mother requires me to do something).

Some items that do not meet the validity requirements (loading factor $<.50$ ) on the permissive mother's parenting variables are in factor I (lack of follow through), namely item number 50 and 52, factor 2 (ignoring misbehavior) which is item number 57, factor 3 (self-confidence), item number 58 and 6I. Statement on item number 50, "Ibu memanjakan saya" (Mother spoils me). Item number 52, "Ibu lebih sering mengancam saya dengan hukuman dari pada yang diberikan" (Mothers threaten me more often with punishment than given). Item number 57, "Ibu percaya diri tentang kemampuannya mengasuh saya" (mother is confident about her ability to care for me). Statement on item number 58 "Ibu melakukan disiplin setelah saya melakukan perilaku salah" (Mother does discipline after I do wrong behavior) and statement 6I. "Ibu saya menetapkan aturan yang ketat untuk saya" (my mother sets strict rules for me).

According to the researchers of this study, the above items did not meet the requirements of validity (loading factor $<.50$ ) due to cultural differences between the cultural origins of the Parenting Style and Dimensions Questionnaire (PSDQ) and 
parenting culture in several regions in Surabaya. Thus, it is necessary to revise and reanalyze the statements.

\section{Conclusion}

Overall, the results of parenting style and dimensions questionnaire (PSDQ) adaptation from Robinson etal (1995) are sufficient to meet the requirements when used as a measurement on adolescents' perceptions of parenting. However, in this study out of 62 items there were ten items that did not meet the validity requirements both on the mother's parenting and on father's parenting. This is due to cultural differences between the origin countries of the parenting style and dimensions questionnaire organization and Indonesian culture. Therefore it is recommended to the next researcher to re-examine by modifying it according to Indonesian culture besides adding the subject and its territory out of Surabaya.

\section{Acknowledgement}

We gratefully acknowledge the support of counseling teachers and participans in several high schools in Surabaya.

\section{References}

Aminayi, M., Chesli, R. C., Shairi, M. R., \& Moharreri, F., (20I5). Comparative study of parenting styles and parenting self-efficacy in mothers of children with and without anxiety symptoms. Fundamentals of Mental Health, 4, 109-191.

Azwar, S. 2012. Reliabilitas dan validitas.Yogyakarta: Pustaka Pelajar

Bornstein, M. H. (20I2). Cultural approaches to parenting. Parent Sci Pract I 2(2-3): $2|2-22|$.

Baumrind, D., Robert E., Larzelere, M \& Elizabeth B. Owens (2010) Effects of Preschool Parents' Power Assertive Patterns and Practices on Adolescent Development,Parenting, I0 (3) I57-20 I

Ferdinand, A (2005). Structural equation modeling dalam penelitian manajemen. Semarang: Badan Penerbit UNDIP. 
Fu, Y., Hou, X., Qin, Q., Meng, H., Xie, P., Huang, Y., Ma, Deng. X. W., Luo, Q., Wang, Y.. Hu, H., Du, L., Haitang Qiu, H., Qiu, T., Li, T. (20I3). Can Parenting Styles and Dimensions Questionnaire (PSDQ) Be Used in China? Psychology, 4(6) 535-540.

Ginsburg, G. S., \& Bronstein, D. (1993). Family factors related to children's intrinsic/extrinsic motivational orientation and academic performance. Child Dev, 64, |46|-|474.

Ghozali, I. (20I I). Aplikasi analisis multivariate dengan program IBM SPSS 19. Semarang: Program Doktor Ilmu Ekonomi Universitas Diponegoro.

Ismaili, E. (20I5), Examining the psychometric properties of the Albanian Version of the Parenting Styles and Dimensions Questionnaire (PSDQ). International Journal of Academic Research in Psychology, 2(I) 23 I 2-I882.

Lansford, J. E., Deater-Deckard, K., Dodge, K. A., Bates, J. E., \&Pettit, G. S. (2004). Ethnic differences in the link between physical discipline and later adolescent externalizing behaviors. Journal of Child Psychology and Psychiatry.45(4): 80 I-8I2.

Luyckx, K.; Tildeley, E.A., Soenens, B., Andrews, J.A., Hampson, S.E., Peterson, M., Duriez, B. (20II). Parenting and trajectories of children's maladaptive behaviors: A 12-year prospective community study. J. Clin. Child Adolesc. Psychol., 40, 468-478.

McMurtry, S. L (20I3). Parenting style differences in Black American and White American Young Adults. Dissertations.The University of Southern Mississippi.

Milevsky, A.; Schlechter, M.; Klem, L.; Kehl, R. (2008).Constellations of maternal and paternal parenting styles in adolescence: Congruity and well-being. Marriage Fam. Rev.44, 8I-98.

Robinson, C. C., Barbara Mandleco, B., Olsen, S. F., \& Hart, C. H. (1995). Authoritative, authoritarian, and permissive parenting practices: development of a new measure. Psychological Reports. 77, 819-830. 